\title{
EDUCACIÓN
}

\section{Satisfacción del estudiante de la carrera de enfermería con la formación recibida en la Universidad María Auxiliadora (UMAX), Año 2014}

\author{
María Lilian Portelli
}

\begin{abstract}
Resumen
Introducción: En los últimos tiempos la educación y, en especial la educación superior, pareciera ocupar el banquillo de los acusados. El aumento casi indiscriminado de instituciones de educación superior junto a la creciente demanda de la matrícula, la heterogeneidad de la misma, la movilidad de estudiantes, académicos y profesionales configuran un escenario lleno de grandes desafíos para este sector. La calidad de la misma es constantemente interpelada por una sociedad que está en constantes y acelerados cambios. En este contexto, las instituciones se ven en la necesidad de redefinir y perfeccionar la calidad del servicio educativo que ofrece, a fin de enfrentar con éxito los retos emergentes y poner en el escenario laboral a profesionales con las competencias requeridas para apostar al desarrollo del país. Para Jiménez González (2011), la satisfacción del estudiante es elemento clave en la valoración de la calidad de la educación, ya que refleja la eficiencia de los servicios académicos y administrativos: su satisfacción con las unidades de aprendizaje, con las interacciones con su profesor y compañeros de clase, así como con las instalaciones y el equipamiento. La visión del estudiante, producto de sus percepciones, expectativas y necesidades, servirá como indicador para el mejoramiento de la gestión y el desarrollo de los programas académicos.
\end{abstract}

Objetivo: Analizar el grado de satisfacción de los estudiantes de la carrera de Enfermería de la Universidad María Auxiliadora, con la formación recibida, en el año 2014.

Material y Método: Este trabajo se enmarcó en un Diseño "No Experimental" pues no hubo manipulación de variables, un "Enfoque "Mixto", de Nivel "Exploratorio-Descriptivo" de corte "Transversal" y ha trabajado con una población total de 47 estudiantes del último año de la Carrera de Enfermería en la UMAX.

Resultados: Los alumnos manifiestan estar satisfechos en la Malla Curricular

1. Universidad María Auxiliadora. Facultad de Ciencias y Humanidades.

El presente trabajo forma parte de un Proyecto de Investigación. Es una Tesis presentada para la obtención del Título de Magíster en Educación, en la Universidad María Auxiliadora que no fue Publicada.

E-mail: lilian-portell@hotmail.com

DOI: 10.26885/rcei.foro.2018.64 


\section{Satisfacción del estudiante de la carrera de enfermería de la UMAX. Portelli}

de la carrera, considerando que un $70 \%$ en la coherencia del Plan, $83 \%$ en la secuencia de asignaturas, 98\% pertinencia de las asignaturas con la realidad, $87 \%$ integración de la teoría con la práctica.

También manifestaron, en su mayoría, estar de acuerdo con la Formación académica del docente, en un $79 \%$ y un $82 \%$ que existe una buena comunicación del docente con el alumnado. El $81 \%$ ha afirmado que existe accesibilidad por parte de las autoridades, el $71 \%$ manifestó que existe buena atención por parte de la secretaría. El 95\% de los mismos afirmó estar satisfecho con las clases teóricas impartidas por los docentes y el $91 \%$ con las clases prácticas. Con relación al sistema Evaluativo, el 65\% afirmó estar de acuerdo. Existe insatisfacción con relación a la infraestructura de la universidad y de todos los estamentos necesarios para poder realizar bien los trabajos, como la biblioteca y los Laboratorios.

Conclusiones: Los alumnos que están culminando el último año de Enfermería en la Universidad María Auxiliadora, tienen un alto nivel de satisfacción con la Malla Curricular de la carrera, también con preparación académica de los docentes y a la metodología de enseñanza mismos, así como al sistema de evaluación implementado. Además, están muy conformes con las orientaciones recibidas de las autoridades de la carrera y del trato recibido de los docentes. Los mismos han manifestado insatisfacción con relación a la infraestructura, la falta de equipamiento de la biblioteca y del laboratorio.

Palabras clave: satisfacción de los estudiantes, formación académica.

\section{Referencias}

Iturralde, G. (2004). La calidad de la educación en la sociedad del conocimiento. Revista Panamericana de Pedagogía, 5.

Jiménez B. (2000). Evaluación de programas, centros y profesores. Madrid: Síntesis.

Jiménez González, A., Terriquez Carrillo, B. \& Robles Zepeda, F. J. (2011). Evaluación de la satisfacción académica de los estudiantes de la Universidad Autónoma de Nayarit. Revista Fuente, 2, 46-56.

Pérez Juste R. (2005). Calidad de la educación, calidad en la educación: hacia su necesaria integración. España: Universidad Nacional de Educación a Distancia. Recuperado en http://www.uned/trabajos

Rodríguez Espinar S. (s/f). La calidad en la enseñanza universitaria (Artículo inédito). Universidad de Barcelona. Recuperado de http://www.uba. es/b15760170.pdf 